\title{
THE “TEU-FEL” PROJECT
}

\author{
G. J. Ernst, ' W. J. Witteman, ${ }^{1}$ J. W. J. Verschunr, ${ }^{1}$ R. F. X. A. M. Mols, ${ }^{1}$ \\ B. M. van Oerle, ${ }^{1}$ A. F. M. Bouman, ${ }^{1}$ J. I. M. Botman, ${ }^{2}$ H. L. Hagedoorn, ${ }^{2}$ \\ J. L. DeLhez ${ }^{2}$ and W. J. G. M. KLEEVEN ${ }^{2}$ \\ 'Department of Applied Physics, University of Twente, P.O. Box 217, 7500 AE Enschede and 'Eindhoven \\ University of Technology, P.O. Box 513, 5600 MB Eindhoven, The Netherlands
}

(Received 30 May 1994)

\begin{abstract}
The free-electron laser of the TEU-FEL project will be based on a $6 \mathrm{MeV}$ photo-cathode linac as injector, a $25 \mathrm{MeV}$ race-track microtron as main accelerator and a hybrid, $25 \mathrm{~mm}$ period undulator. The project will be carried out in two phases. In phase one only the $6 \mathrm{MeV}$ linac will be used, The FEL will then produce tunable radiation around $200 \mu \mathrm{m}$. In phase two the linac will be used as an injector for the microtron. The FEL will then produce tunable radiation around $10 \mu \mathrm{m}$. Technical information will be presented on the different subsystems.
\end{abstract}

\section{INTRODUCTION}

TEU-FEL stands for a cooperation between Twente University, Eindhoven Technological University and Ultra Centrifuge Nederland. The aim of the project is to build a free electron laser (FEL) and contribute to the understanding and technological development of FEL's. This FEL will consist of a photo-cathode linac which will accelerate the electrons up to $6 \mathrm{MeV}$, a race-track microtron which will accelerate the electrons up to $25 \mathrm{MeV}$, a hybrid undulator, an electron bending system and an optical cavity.

The project will be carried out in two phases. In phase I an FEL will be built with the photo-cathode linac and the hybrid undulator only. No optical cavity will be used: the electrons will be focused directly into the undulator so that the FEL will work as an amplifier of spontaneous emission or, eventually, as an amplifier of an injected signal. In phase II the race-track microtron will be used and the undulator will be placed inside an optical cavity and then, of course, an electron bending system will be required. The main characteristics of the project are summarized in Table 1.

Free Electron lasers require very good quality electron beams. That is why we have chosen for a photo-cathode linac instead of a classical injector with a thermionic gun. A photo-cathode linac can produce almost an order of magnitude higher peak current which provides a much higher gain. Also the emittance is almost an order of magnitude lower so that shorter wavelengths can be obtained. On the other hand such a system is more complicated and not as well developed. It also requires a drive laser. The choice for a Race-Track Microtron as main accelerator is because such an accelerator has a stable phase area. This results in a lower energy spread and thus higher gain. Another advantage is its high efficiency with respect to the required $R F$ power. On the other hand the current carrying capability is not as high as for a linac. How high is not known yet, but it probably is about an order of magnitude lower. We did not obtain FEL radiation yet, but we will present here the status of the different parts of our projects. Let me first give an overview of the total system.

A schematic overview can be seen in Fig. 1. We start with a central driver with a $\mathrm{mW}$ level at $40.625 \mathrm{MHz}$. This driver delivers the signal for the mode-locker of the Nd:YLF laser as well as, after frequency multiplication to $1.3 \mathrm{GHz}$, for the photo-cathode linac. In this way automatic frequency synchronization is obtained between the Nd:YLF laser and the accelerator. This linac 
Table 1. Main characteristics of the TEUFEL project

\begin{tabular}{lll}
\hline & Phase I & Phase II \\
\hline Accelerator & Linac & Linac + RT-microtron \\
Accelerator energy & $6 \mathrm{MeV}$ & $25 \mathrm{MeV}$ \\
$R F$-frequency & $1.3 \mathrm{GHz}$ & $1.3 \mathrm{GHz}$ \\
Micropulse duration & $20 \mathrm{ps}$ & $35 \mathrm{ps}$ \\
Micropulse repetition frequency & $81.25 \mathrm{MHz}$ & $81.25 \mathrm{MHz}$ \\
Peak current & $350 \mathrm{~A}$ & $100 \mathrm{~A}$ \\
Macropulse duration & $15 \mu \mathrm{s}$ & $15 \mu \mathrm{s}$ \\
Pulse repetition frequency & $10 \mathrm{~Hz}$ & $10 \mathrm{~Hz}$ \\
Undulator period & $25 \mathrm{~mm}$ & $25 \mathrm{~mm}$ \\
Number of undulatory periods & 50 & 40 \\
$K$-value & 1 & 1 \\
Undulator gap & $8 \mathrm{~mm}$ & $8 \mathrm{~mm}$ \\
Radiation wavelength & $180 \mu \mathrm{m}$ & $10 \mu \mathrm{m}$ \\
Optical cavity length & - & $1.85 \mathrm{~m}$ \\
\hline
\end{tabular}

requires a power of about $5 \mathrm{MW}$, so a number of amplification stages is required to bring the central driver signal to that level.

The whole system is a pulsed one with a repetition frequency of $10 \mathrm{~Hz}$ maximum and a pulse duration of $20 \mu \mathrm{s}$. Each pulse consists of a train of micropulses about $20 \mathrm{ps}$ long and with a repetition frequency of $81.25 \mathrm{MHz}$ being the 16th subharmonic of $1.3 \mathrm{GHz}$.

We will now discuss the several parts of our FEL.

\section{PERFORMANCE AND STATUS OF THE SUBSYSTEMS}

The linear accelerator

The $6 \mathrm{MeV}$ linear accelerator is the main accelerator in the phase I experiment and the preaccelerator in the phase II experiment. It has been designed and constructed at Los Alamos National Laboratory. It is a five and a half cell linac and an improved version of their own linac used to upgrade HIBAF. It is an on-axis-coupled linac mainly because such a structure is more compact than a side-coupled one. This enables the construction of a more compact solenoid and bucking coil. In the first half cell the photo-cathode is placed where the electrons are generated.

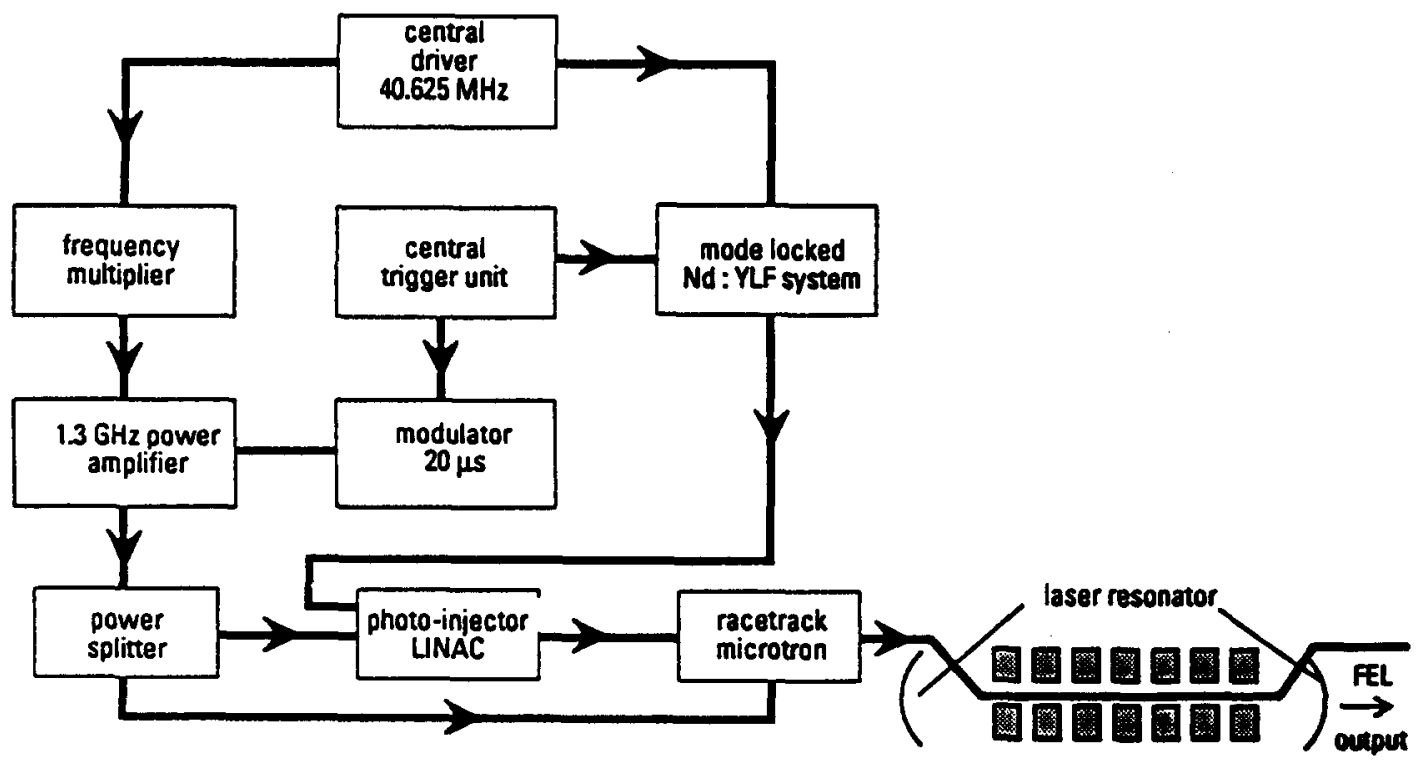

undulator

Fig. 1. Schematic layout of the TEU-FEL. 
Due to the high accelerating field strength at the photo-cathode surface, emittance growth due to space charge is reduced. Also the noses in the first one and a half cells are optimized for low emittance growth. Because $\mathrm{CsK}_{2} \mathrm{Sb}$ will be used as photo-cathode material, ultra-high vacuum $\left(\sim 10^{-10} \mathrm{Torr}\right)$ is required. That is why the structure can be baked out at $350^{\circ} \mathrm{C}$ and the pumping speed is increased by a large number of pumping slots in each cell.

The radial dependence of the accelerating field as well as the space-charge forces cause a defocussing of the electron beam. The solenoid field has to compensate this defocussing effect. A bucking field is present to make the magnetic field zero at the cathode surface. Figure 2 shows a preliminary emittance measurement at $70 \mathrm{~A}$ peak current. This figure shows a so-called quadrupole scan: the spot-size of the beam in one direction is measured for different focusing strengths for a fixed screen position. The unnormalized FWHM emittance can be found by using the following formula:

$$
x=x_{\min } \sqrt{1+\left(\frac{L}{x_{\min }}\right)^{4}\left(\frac{\epsilon_{\mathrm{un}}}{f w}\right)^{2}\left(1-\frac{f \omega}{f}\right)^{2}},
$$

where

$x=$ measured spotsize,

$x_{\min }=$ minimum spotsize,

$L=$ spacing between quadrupole and screen,

$f=$ focal length of quadrupole,

$f w=$ focal length of quadrupole producing the minimum spotsize,

$\epsilon_{\mathrm{un}}=$ unnormalized FWHM emittance.

From the minimum spotsize and the shape of the curve we found an unnormalized FWHM emittance of $1.7 \pi \mathrm{mm} \mathrm{mrad}$, which means a normalized RMS emittance of $4 \pi \mathrm{mm} \mathrm{mrad}$.

\section{$N d: Y L F$ laser-amplifier system}

This laser has to illuminate the photo-cathode. A big advantage of using a laser-photo-cathode system is that the required timestructure of the electron beam can be produced directly by giving

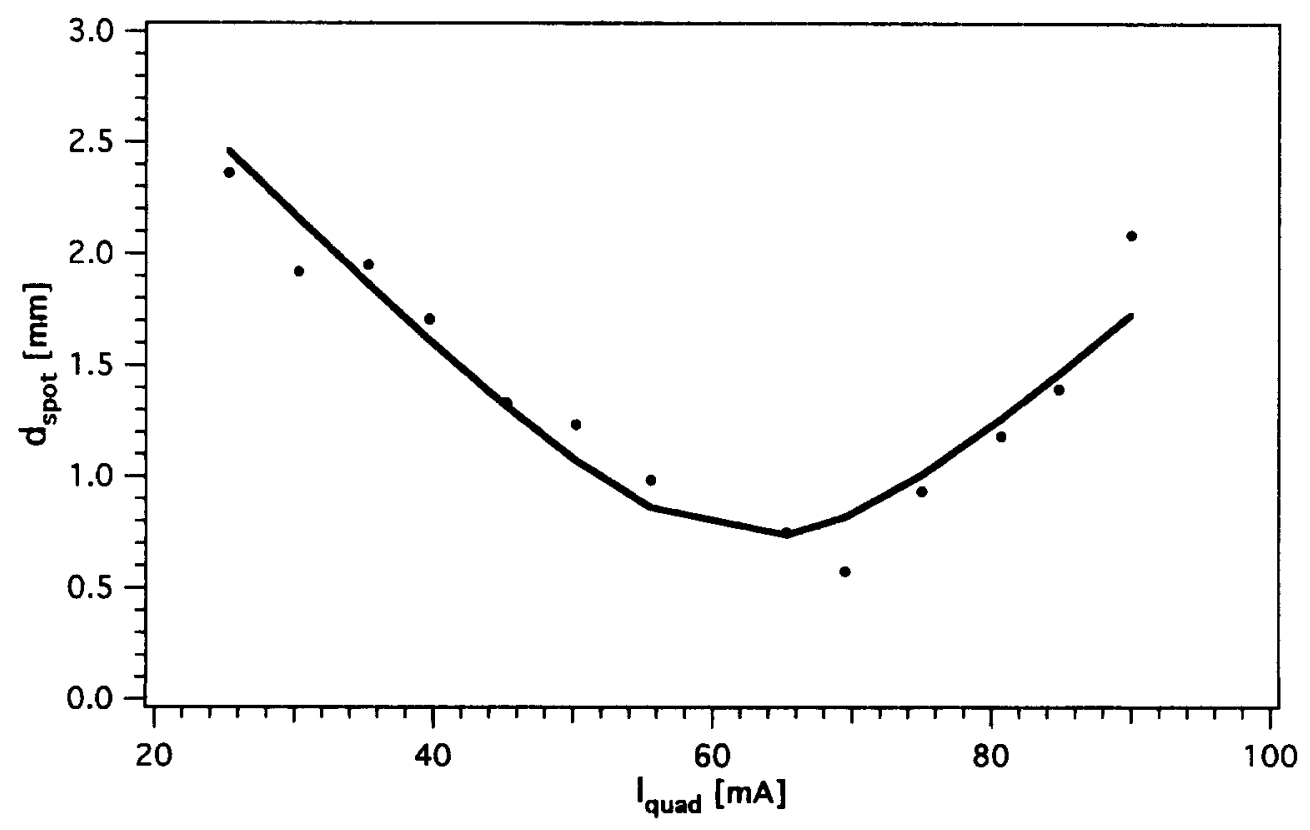

Fig. 2. A quadrupole scan of the electron beam for $70 \mathrm{~A}$ peak current. 
Table 2. Main drive laser requirements

\begin{tabular}{lc}
\hline Macropulse time & $15 \mu \mathrm{s}$ \\
Repetition frequency & $10 \mathrm{~Hz}$ \\
Micropulse time & $20 \mathrm{ps}$ \\
Wavelength & $527 \mathrm{~nm}$ \\
Energy per micropulse & $5 \mu \mathrm{J}$ \\
Amplitude stability & $<1 \%$ \\
Phase stability & $<1 \mathrm{ps}$ \\
\hline
\end{tabular}

Table 3. Parameters of the pulse compressor

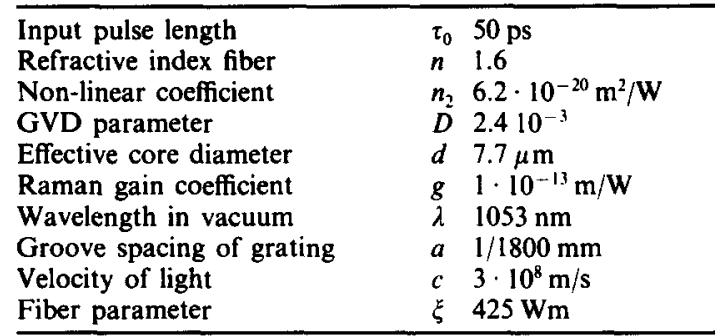

the output radiation that required time structure. The requirements for the laser can be found in Table 2.

In order to fulfil the laser requirements we have chosen for a frequency doubled Nd:YLF laser-amplification system. A schematic of this laser can be seen in Fig. 3. In principle it exists of a mode-locked Nd:YLF laser with a mode lock frequency of $81.25 \mathrm{MHz}$. It produces a continuous train of pulses about $50 \mathrm{ps}$ long. A pulse compressor compresses the pulses down to $20 \mathrm{ps}$. Then an acoustic-optic modulator slices out of the continuous train $10 \mu \mathrm{s}$ long pulses. The power in one $\mu$-pulse after the slicer is $0.7 \mathrm{~nJ}$. This is amplified in two double pass amplification stages to a level of $15 \mu \mathrm{J}$ per $\mu$-pulse.

Finally frequency doubling in an LBO crystal, giving $40 \%$ conversion efficiency, results in $6 \mu \mathrm{J}$ of green light $(\lambda=0.5265 \mu \mathrm{m})$.

The pulse compressor set-up is shown in Fig. 4. It is a conventional grating-prism arrangement. Table 3 shows the various parameters of the compressor. Figure 5 shows the measurements of the compression factor, which is the ratio between the original and final pulse length, as a function of the average laser power for different fiber lengths. For all the measurements the grating prism distance has been optimized for maximum compression. As can be seen from the figure the compression factor saturates for all fiber lengths. This has to do with the onset of stimulated Raman Scattering. It is interesting to look at the autocorrelation signal for the same compression factor (about 10) but different fiber length. This is shown in Fig. 6. The substructure for 10,25 and $50 \mathrm{~m}$ is almost the same. For $100 \mathrm{~m}$ fiber length the substructure begins to decrease because of the effect of group velocity dispersion. Because we needed a compression factor of about four our choice was a $10 \mathrm{~m}$ long fiber because the average power and thus the peak power was a maximum for that length.

Amplitude as well as phase jitter is a well-known problem of drive lasers for photo-cathode linacs. We performed amplitude and phase jitter measurements before and after the pulse compressor. In that way we could find out what is the jitter of our Antares Nd:YLF mode-locked laser and what is the contribution of the pulse compressor. The method we used has been described

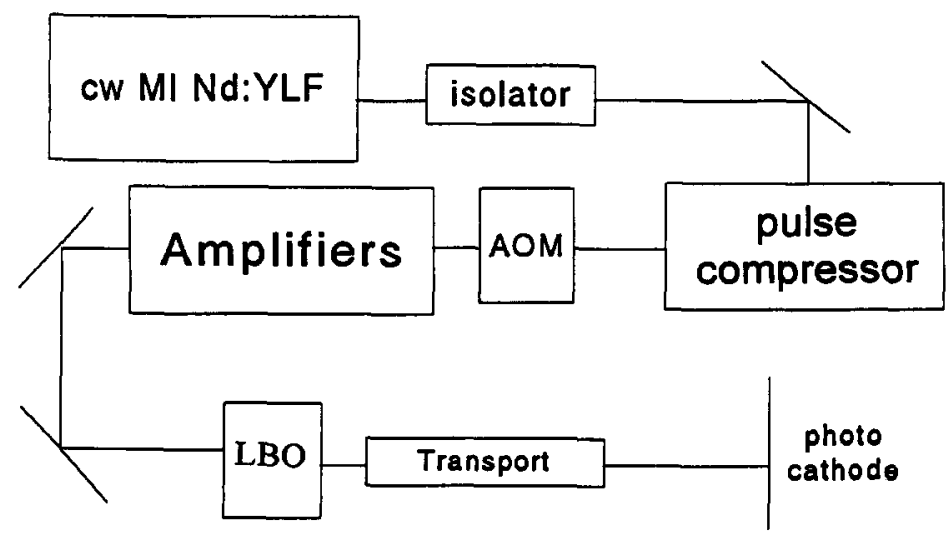

Fig. 3. Schematic of Nd:YLF laser-amplifier system. 


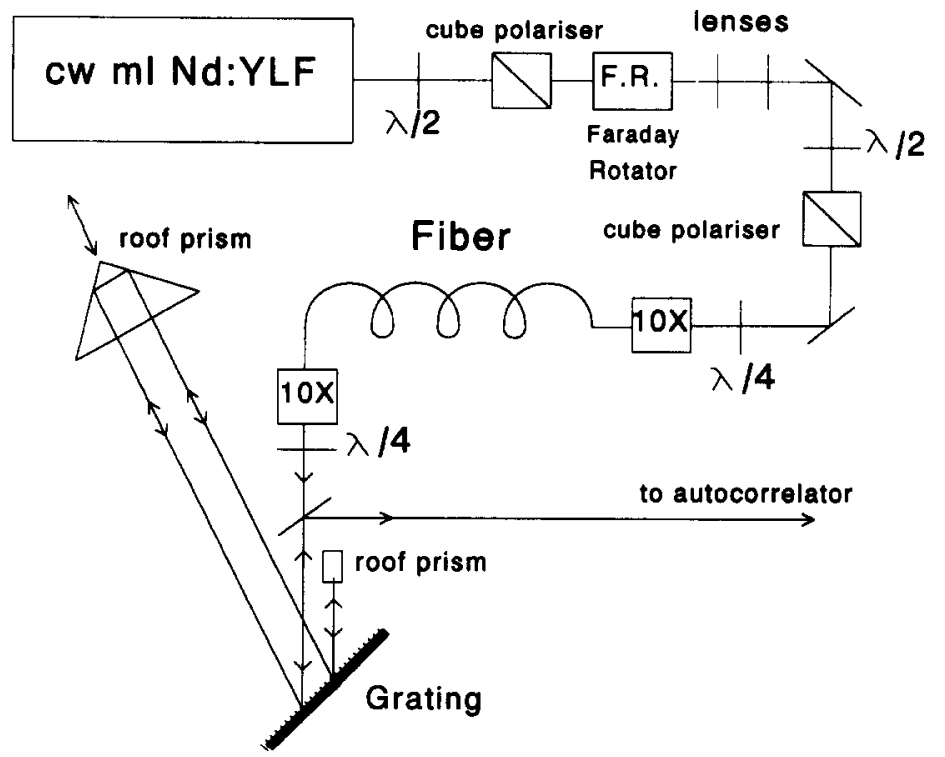

Fig. 4. Pulse compressor setup.

by von der Linde in his beautiful paper. ${ }^{(1)}$ He showed that amplitude (= energy) and phase (= time) jitter can be measured from the spectral content of the laser power around the different harmonics of the mode-lock frequency. When no jitter is present very sharp peaks should be found. Amplitude jitter gives a broadening of the peak independent of the harmonic number and phase jitter gives a broadening that goes quadratic with the harmonic number. The relations are

$$
\begin{gathered}
\langle A\rangle^{2}=\left(\frac{P_{C}}{P_{A}}\right)_{N} \frac{\Delta f_{A}}{\Delta f_{R B}} \\
\langle 2 \pi N J\rangle^{2}=\left(\frac{P_{B}}{P_{A}}\right)_{N} \frac{\Delta f_{j}}{\Delta f_{R B}}
\end{gathered}
$$

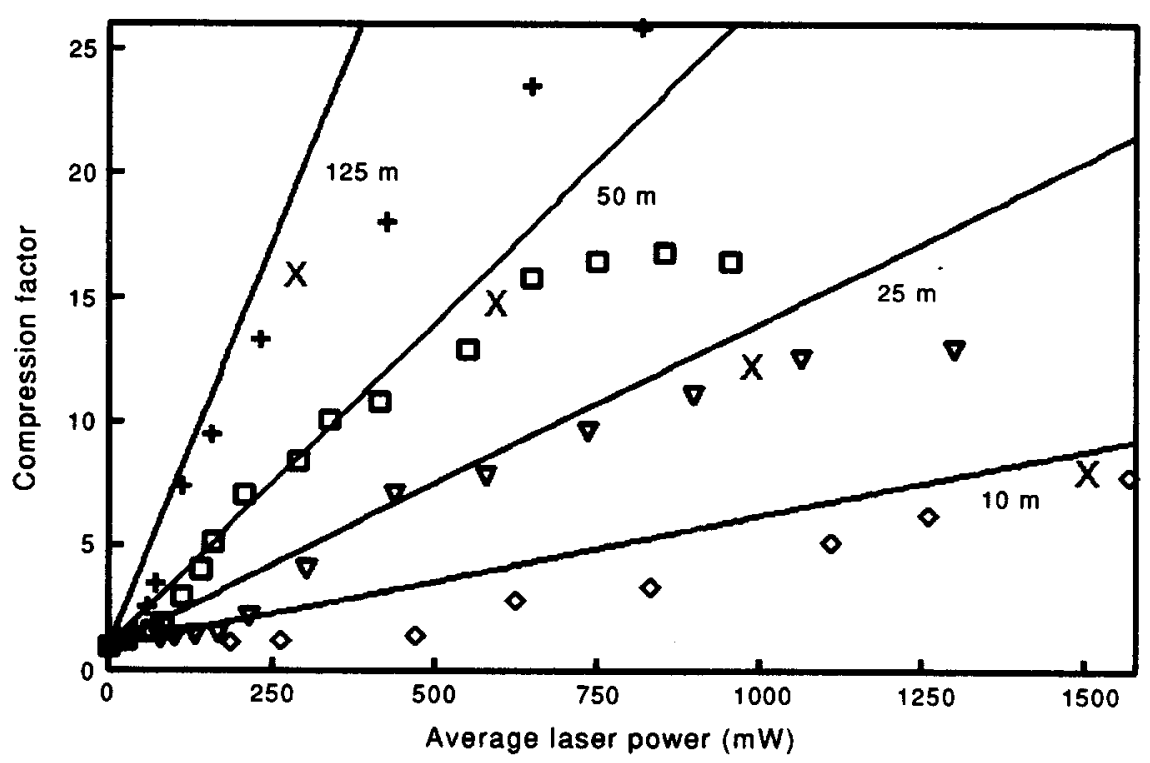

Fig. 5. The compression factor as a function of the average laser power for various fiber lengths. The straight lines represent theoretical predictions. The points indicated by X represent the power where SRS starts to influence the compression factor. 


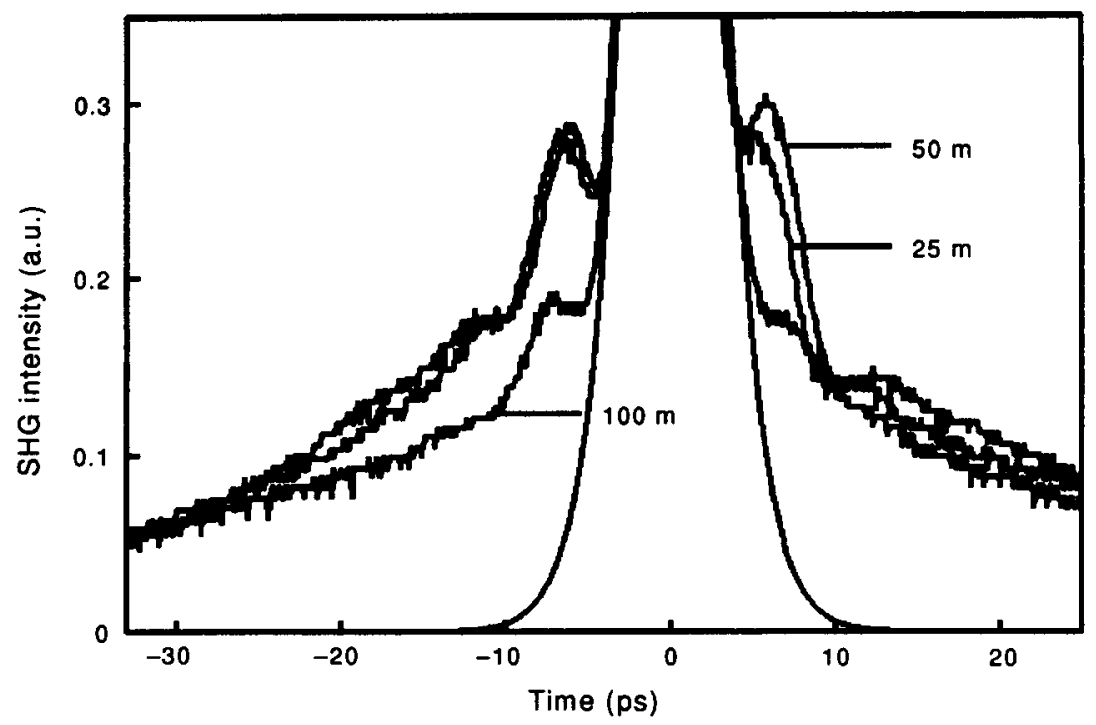

Fig. 6. For the case of equal compression factors ( $\sim 10$ times) the substructure of the pulse is shown for fiber length of 25,50 and $100 \mathrm{~m}$. The theoretical $\mathrm{sech}^{2}$ function is also shown for comparison.

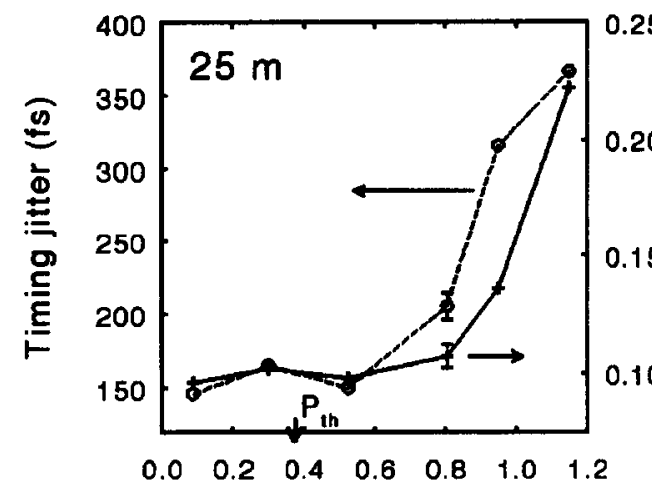

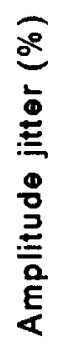

Input power (W)

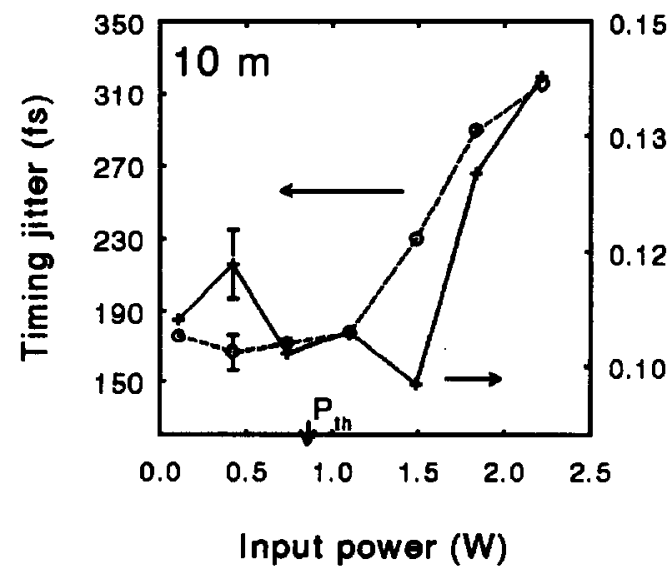

送

Fig. 7. The amplitude and phase jitter measured as a function of the input power for a $10 \mathrm{~m}$ fiber (top) and a $25 \mathrm{~m}$ fiber. A fast increase of the jitter is measured above the threshold for SRS. 
Table 4. Parameters of three important laser materials

\begin{tabular}{lcccc}
\hline & Nd:YLF & Nd:YLF & & \\
& $\sigma$ & $\pi$ & Nd:Glass & Nd:YAG \\
\hline$\lambda\left({ }^{4} \mathrm{~F}_{3 / 2} \rightarrow{ }^{4} \mathrm{I}_{1 / 2}\right)(\mathrm{nm})$ & 1053 & 1047 & 1053 & 1064 \\
$\sigma\left(10^{-19} \mathrm{~cm}^{2}\right)$ & 1.2 & 1.8 & 0.44 & 6.5 \\
$\tau(\mu \mathrm{S})$ & 480 & 480 & 357 & 230 \\
$\Delta \lambda(\mathrm{nm})$ & 1.35 & - & 21.2 & 0.45 \\
$E_{\mathrm{S}}\left(\mathrm{J} / \mathrm{cm}^{2}\right)$ & 1.5 & 1.0 & 4.3 & 0.3 \\
\hline
\end{tabular}

where

$\langle A\rangle$ and $\langle J\rangle=$ the r.m.s. value of the amplitude and phase jitter, respectively,

$\Delta f_{A}$ and $\Delta f_{j}=$ band width of the amplitude and phase signals, respectively

$\Delta f_{R B}=$ the resolution bandwidth of the spectrum analyser

$P_{A}, P_{B}$ and $P_{C}=$ the power levels of the sharp peak, the phase signal and the amplitude signal, respectively.

In this way the jitter of the Antares Nd:YLF laser has been determined. The r.m.s. amplitude jitter appeared to be $0.1 \%$ and the r.m.s. phase jitter $170 \mathrm{fs}$.

Also measurements have been performed after the pulse compression. The results are shown for a 10 and $25 \mathrm{~m}$ fiber in Fig. 7. These figures clearly show that a pulse compressor does not contribute to the amplitude or phase jitter as long as the power level remains below the threshold for stimulated Raman scattering.

Once the choice has been made for the oscillator namely Nd:YLF there are two possible materials for the amplifiers: Nd:YLF and Nd:phosphate glass. The relevant physical parameters are shown in Table 4. For comparison also the figures of Nd:YAG are shown. From the figures a second reason, apart from thermal instability, can be seen why Nd:YAG is not suitable for us: the bandwidth of the transition is too small to amplify 20 ps pulses. Nd:YLF is non-isotropic. The wavelength for amplification is dependent on the direction of polarization. An advantage of this material is (compared with $\mathrm{Nd}$ :glass) its higher gain and its possibility to be used at high repetition rate $(\sim 20 \mathrm{~Hz})$.
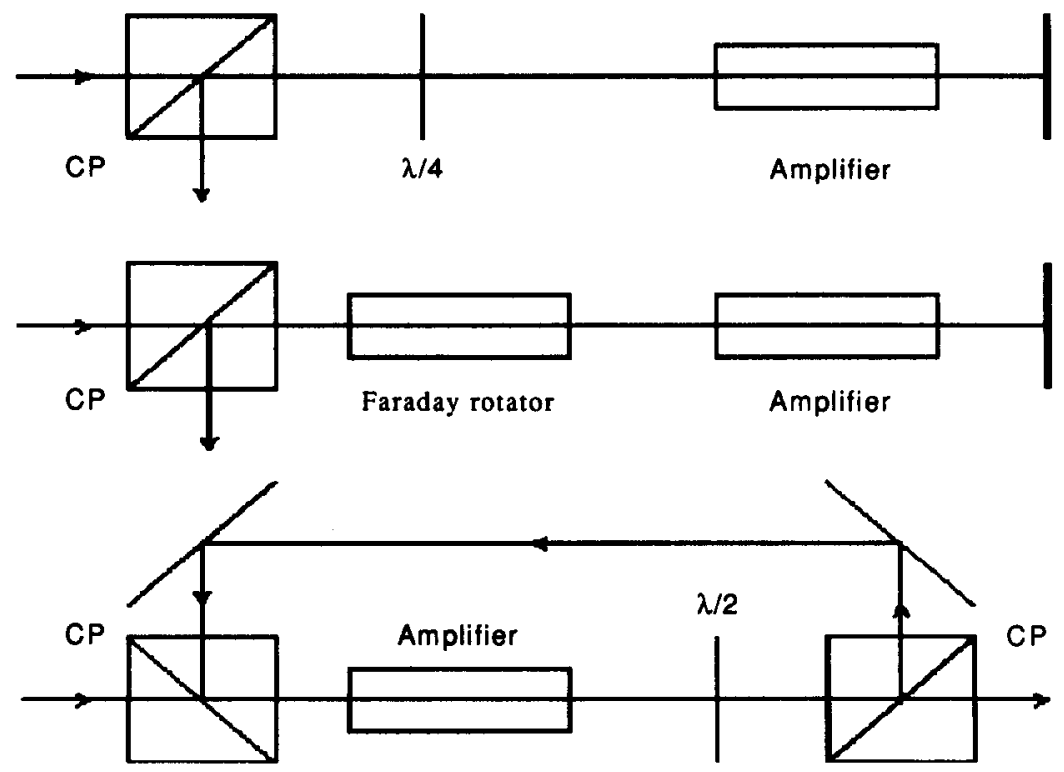

Fig. 8. The three possible configurations for a double pass amplifier setup where $\mathrm{CP}$ is a cube polarizer and $\lambda / 2$ and $\lambda / 4$ are, respectively, a half and a quarter wave plate. 


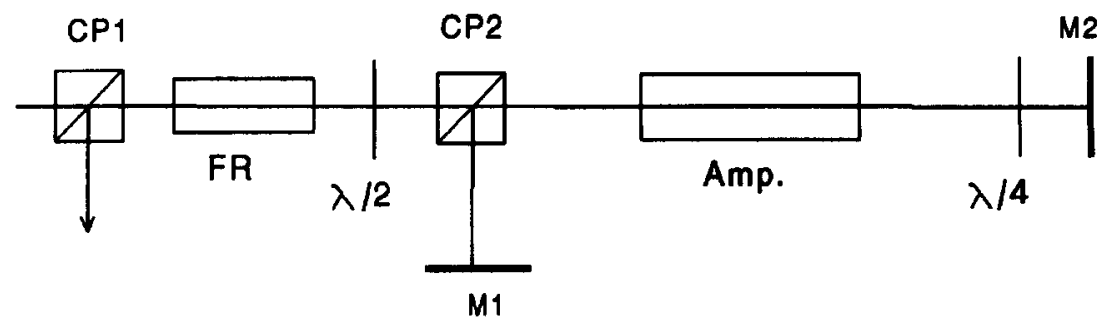

Fig. 9. Lay out of the four-pass amplifier. The seed beam enters from the left. The meaning of the various symbols are: CP, cube polarizer; FR, Faraday rotator; Amp, amplifier; M, mirror.

The output level of each micropulse after the pulse compressor is of the order of $1 \mathrm{~nJ}$. In order to produce the required $350 \mathrm{~A}$ peak current at $20 \mathrm{ps,} \mathrm{which} \mathrm{makes} 7 \mathrm{nC}$ of total charge, from a $1 \%$ quantum efficiency photo-cathode, an energy of $3 \mu \mathrm{J}$ at the second harmonic is required. Suppose that an efficiency of $30 \%$ can be obtained for producing the second harmonic frequency, than about $10 \mu \mathrm{J}$ per micropulse of IR energy is required. This means an amplification of 10.000 . So a multipass amplification configuration has to be used because the amplification in one pass through an amplifier is much too small. Because high spatial quality is required only an on-axis configuration is possible, where the forward and backward travelling beams are separated by rotation of the plane of polarization.

The three possible configurations are shown in Fig. 8, where separation of the two beams is accomplished in a cube polarizer by rotation of the plane of polarization over $90^{\circ}$. It is also possible to use a four-pass configuration shown in Fig. 9. This configuration is especially of interest for low gain amplifiers or very low input signals.

A four-pass amplifier has been built with $\mathrm{Nd}$ :glass as the active medium. The results can be seen in Fig. 10. The total gain for a $10 \mathrm{~cm}$ long crystal was about 20 which is too small to be of practical use for us. So we decided to build two double pass amplifies with Nd:YLF as active medium. The first one has a rod diameter of $6 \mathrm{~mm}$ and the second one of $7 \mathrm{~mm}$. The measured single pass gain is 50 and 20 respectively. So the total gain can be as high as $10^{6}$. In practice the gain is lower due to losses in the system and due to self amplified spontaneous emission. A very difficult problem in building such a system is to circumvent self oscillation. A minor reflection on one of the components can cause this effect. It took us a long time to solve this problem.

A requirement for the free electron laser is that the macropulse has to be flat in time. So all the micropulses have to contain the same amount of energy. As long as we are in the small signal gain regime there is no problem. Our macropulse is $10 \mu \mathrm{s}$ long whereas the gain pulse of the amplifiers

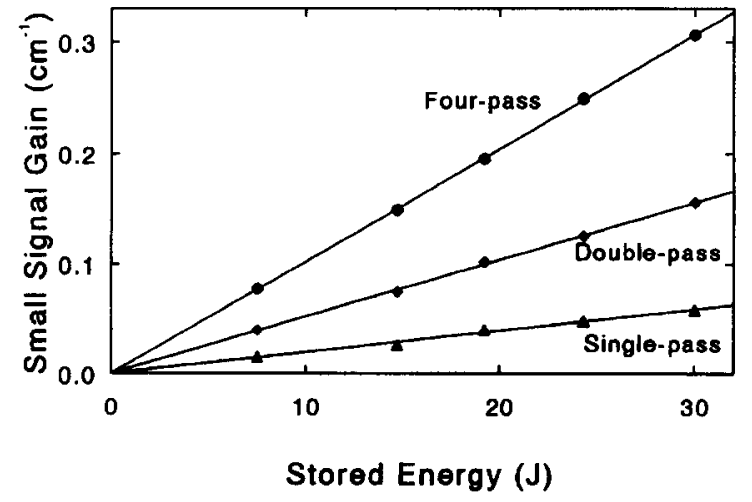

Fig. 10. The measured small signal gain for a single-pass, double-pass and four-pass amplification scheme for Nd:glass. The small-signal gain is measured as a function of the stored energy in the flash lamp of the amplifier. 
Table 5. The characteristic parameters of the materials of the materials KTP and LBO, used for second harmonic generation

\begin{tabular}{lcc}
\hline & KTP & LBO \\
\hline$d_{\text {eff }}(\mathrm{pm} / \mathrm{V})$ & 3.0 & 1.0 \\
Damage threshold 1 ns pulse $\left(\mathrm{GW} / \mathrm{cm}^{2}\right)$ & 0.4 & 20 \\
Walk-off ( ${ }^{\circ}$ & 0.33 & 0 \\
Acceptance angle (mrad cm) & 13.57 & - \\
Group velocity mismatch $(\mathrm{ps} / \mathrm{cm})$ & 4.39 & 0.56 \\
Spectral acceptance bandwidth $(\mathrm{nm} \mathrm{cm})$ & 0.3 & 1.6 \\
Bandwidth for 10 ps pulses (nm) & 0.16 & 0.16 \\
Aperture length (mm) & 24 & - \\
Quasi static interaction length (mm) & 23 & 179 \\
Spectral acceptance length (mm) & 19 & 100 \\
\hline
\end{tabular}

is several hundreds of microseconds. So injection at the top of the gain pulse will be sufficient. However as soon as saturation plays a role, the amplification of the first micropulse will be largest and subsequent micropulses will have lower and lower gain resulting in a droop of the micropulse energy over the macropulse. The solution to this problem is that there has to be a balance between the gain of a micropulse and the production of inversion between two micropulses. This situation can be reached by a proper timing of the injection pulse with respect to the pump pulse of the amplifier. In this way we obtained a macropulse, flat within a few per cent and with an energy of $12 \mu \mathrm{J}$ per micropulse.

Frequency doubling has been obtained in $\mathrm{KTiOPO}_{4}(\mathrm{KTP})$ and $\mathrm{LiB}_{3} \mathrm{O}_{5}(\mathrm{LBO})$. The characteristics of both materials are given in Table 5 .

LBO can be used under the non-critical phase matching condition. The limiting crystal length of LBO is the maximum available length of $18 \mathrm{~mm}$. For KTP a $10 \mathrm{~mm}$ long crystal has been used. The measurements for both crystals can be seen in Fig. 11. A maximum conversion efficiency of $40 \%$ has been obtained.

\section{Photo-cathodes}

$\mathrm{Up}$ to now we used $\mathrm{K}_{3} \mathrm{Sb}$ and $\mathrm{CsK}_{2} \mathrm{Sb}$ as photo-cathode material. The cathode is placed on an actuator which is used to move the cathode into the first half cell of the linac. The containers with the evaporation materials are mounted on another actuator perpendicular to the first one. In order to evaporate a certain material onto the cathode the respective container has to be moved in front of the cathode. During fabrication the cathode is kept on a potential of about $100 \mathrm{~V}$, so it is

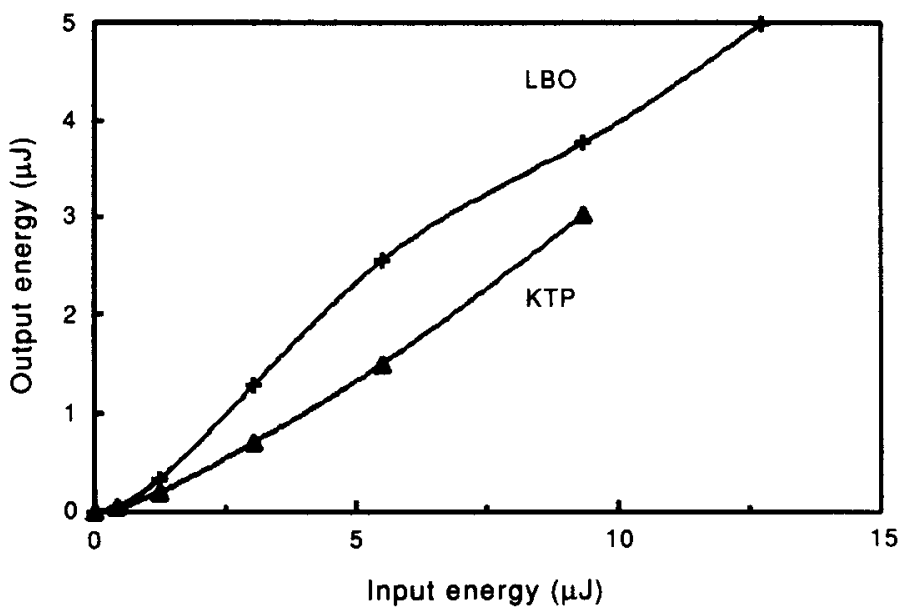

Fig. 11. The measured energy per micropulse of green light after frequency doubling using KTP and LBO for different input powers. The beam waist was $90 \mu \mathrm{m}$. 


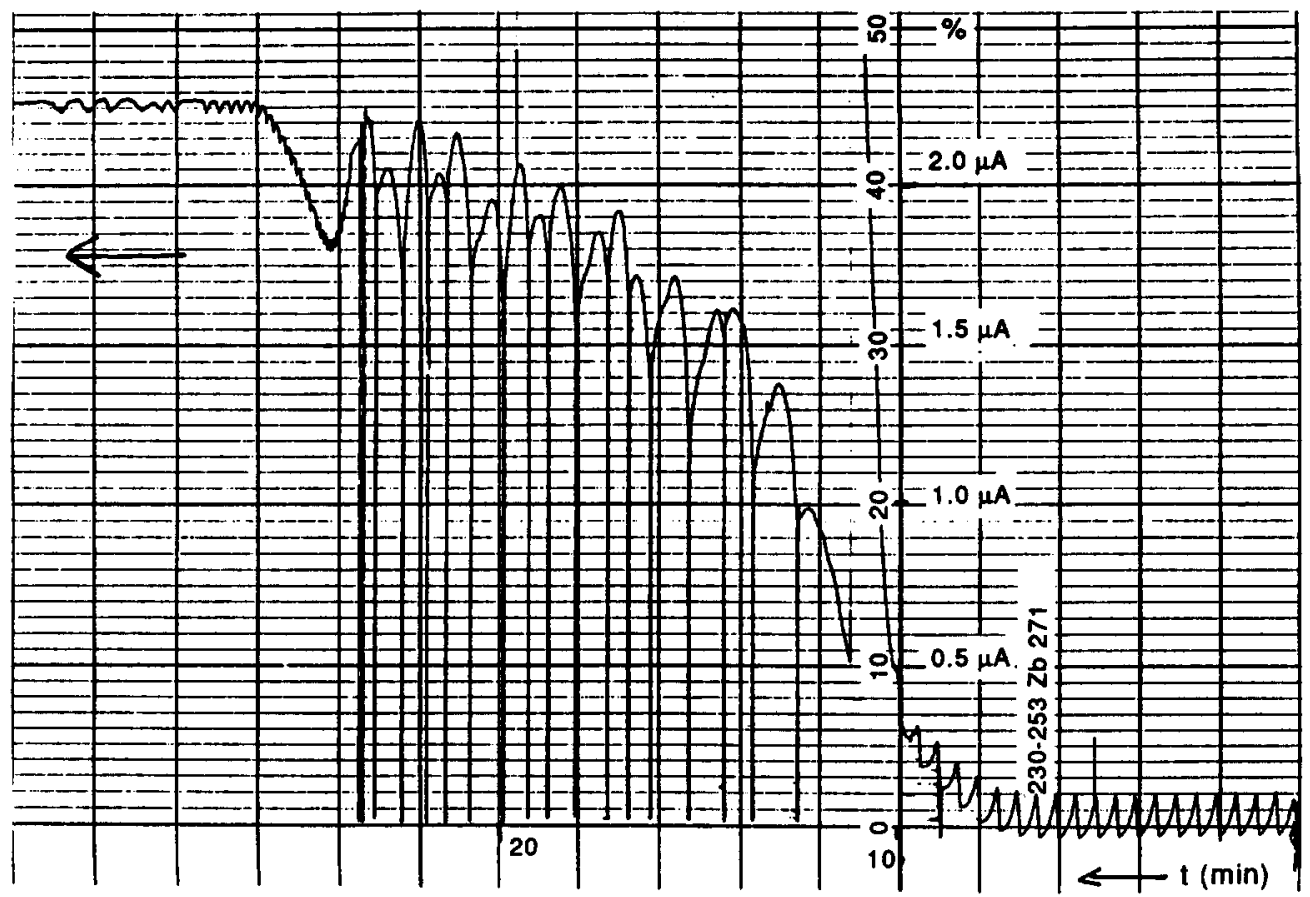

Fig. 12. Photo current during fabrication of a multi-layer $\mathrm{K}_{3} \mathrm{Sb}$ photo cathode.

possible, by illumination with a $1.5 \mathrm{~mW}$ green $\mathrm{HeNe}$ laser, to monitor the quantum-efficiency during the preparation process. A typical example of such a process is shown in Fig. 12. Starting with a layer of $\mathrm{Sb}$, alternating layers of $\mathrm{K}$ and $\mathrm{Sb}$ are evaporated until a maximum in photo current has been reached. After that the $\mathrm{CsK}_{2} \mathrm{Sb}$ photo-cathode is completed by adding a layer of Cs (not shown in the figure). Although we tried to standardize the preparation process as well as possible there is always a large variation in the $\mathrm{QE}$ of different cathodes. Figure 13 shows this variation for $\mathrm{K}_{3} \mathrm{Sb}$ as well as $\mathrm{CsK}_{2} \mathrm{Sb}$ photo-cathodes. The lifetime of the photo-cathodes is an important point. As long as the cathodes remains in the preparation chamber where the vacuum is very good (about $4.10^{-10}$ Torr) the lifetime is very long (many days). However under operation conditions in

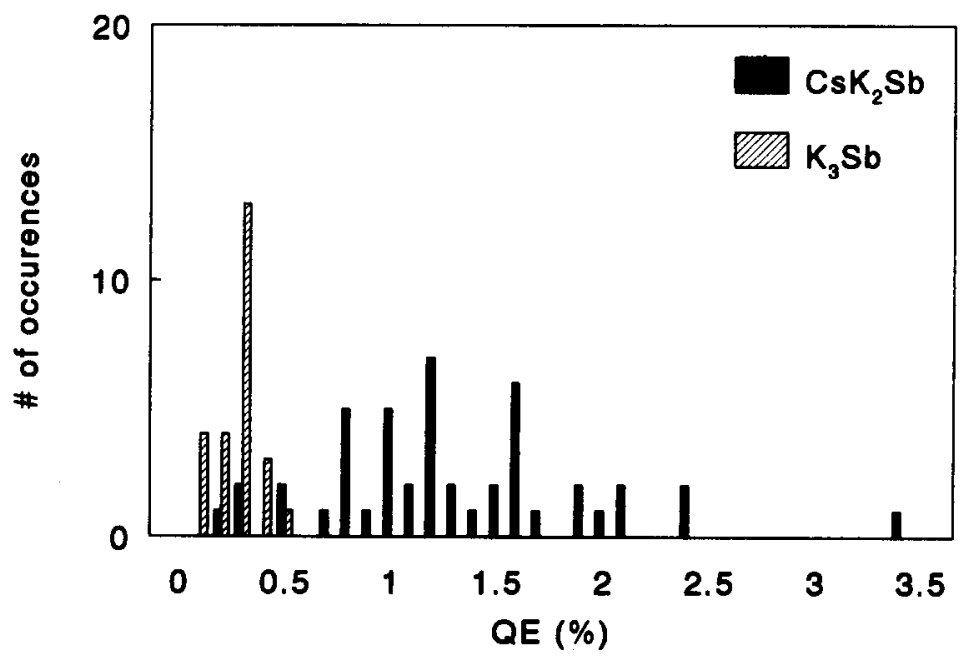

Fig. 13. Statistics of $\mathrm{K}_{3} \mathrm{Sb}$ (dashed bars) and $\mathrm{CsK}_{2} \mathrm{Sb}$ (solid bars) photo cathodes between September 1993 and March 1994. 
the linac the lifetime is 1 or $2 \mathrm{~h}$ only. Figure 14 shows the $\mathrm{QE}$ for different wavelengths of our cathodes under different conditions. As can be seen it is possible to enhance the quantum efficiency again after use by adding a layer of Cs. It can be seen that the lifetime is longer for shorter wavelength. This suggest a drive laser operating at the third and fourth harmonic frequency.

\section{The undulator}

The undulator is of the planar hybrid type, i.e. alternating permanent magnetic material and poles. As magnetic material $\mathrm{SmCo}_{5}$ has been used and the poles are made from vanadium permendur. The undulator has a wavelength of $25 \mathrm{~mm}$, a $K$ value of 1 (which means a magnetic field strength on axis of $0.7 \mathrm{~T}$ ) and equal focusing in both transverse directions due to a circular shape of the poles. A quarter period of the undulator is shown in Fig. 15. The shape of the poles as well as the magnetic material has been optimized with respect to the harmonic content of the field and the field strength itself. The design parameters are shown in Table 6.

In order to keep the tolerances of all periods as small as possible each magnet has been built out of six smaller ones. Each of those small magnets have been characterized in a Helmholz coil configuration, sorted and combined into groups of six with a small tolerance. Each group of six has been glued together with a pole in a keeper. Finally the keepers are mounted on a rigid bench.

The field the completed undulator has been measured with the so-called pulsed wire method. In this method a wire is mounted inside the undulator. By sending a large current $(100 \mathrm{~A})$ through this wire, the wire moves vertically under the influence of the Lorentz force. This movement propagates with the speed of sound in the longitudinal direction, By recording the displacement of the wire at a certain position outside the magnetic field region as a function of time, information can be obtained on the relative magnitude of the magnetic field as a function of position. By using a short current pulse the first integral as a function of position is obtained. By using a long pulse the second integral, which gives information on steering errors of the propagating electron beam,

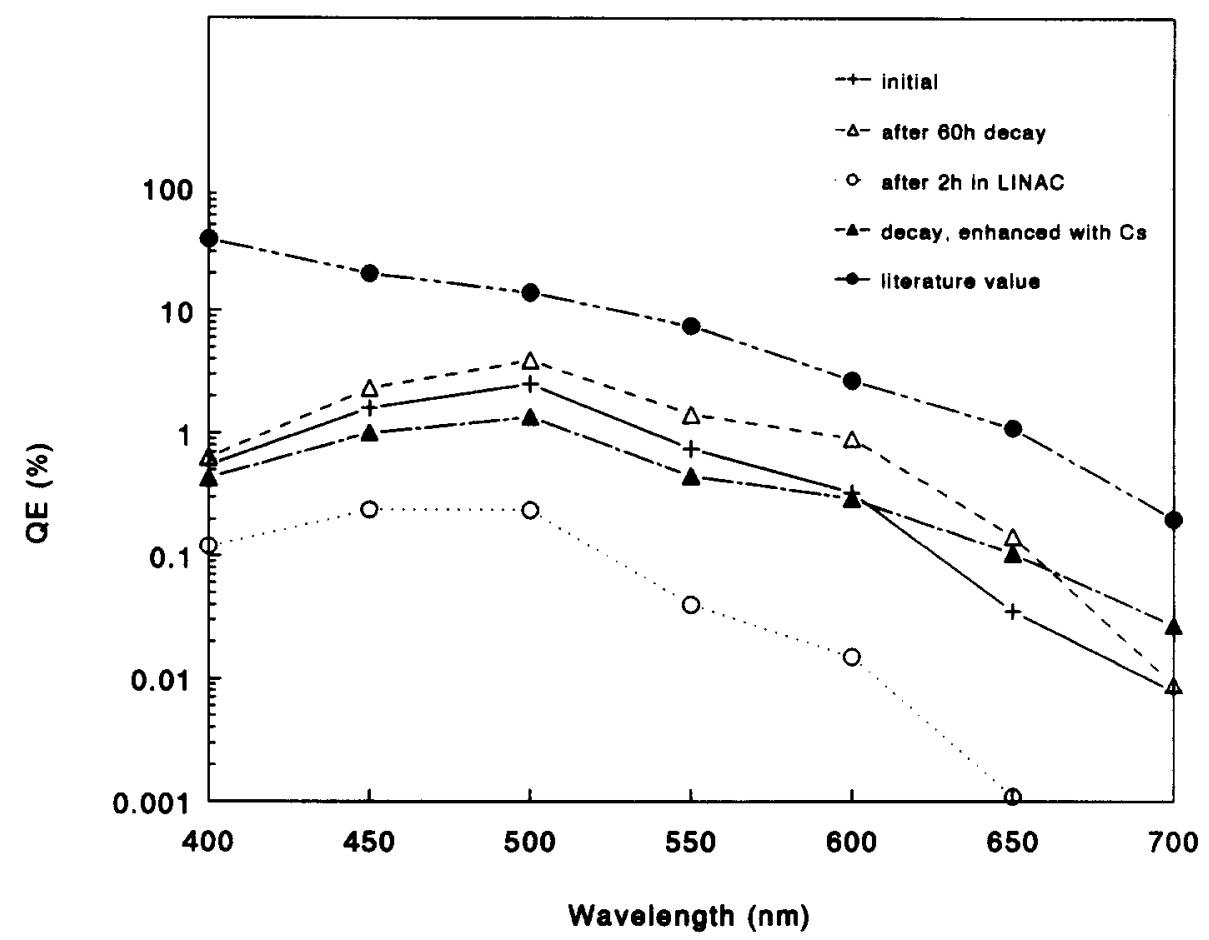

Fig. 14. Spectral sensitivity of $\mathrm{CsK}_{2}$ Sb photo cathodes. $\mathrm{X}$, initial values; $\triangle$, after $60 \mathrm{~h}$ rest in preparation chamber; $O$, after $2 \mathrm{~h}$ in use in linac, $\Delta$, after enhancement with $\mathrm{Cs} ; \boldsymbol{O}$, as measured by Sommer. ${ }^{(2)}$ 


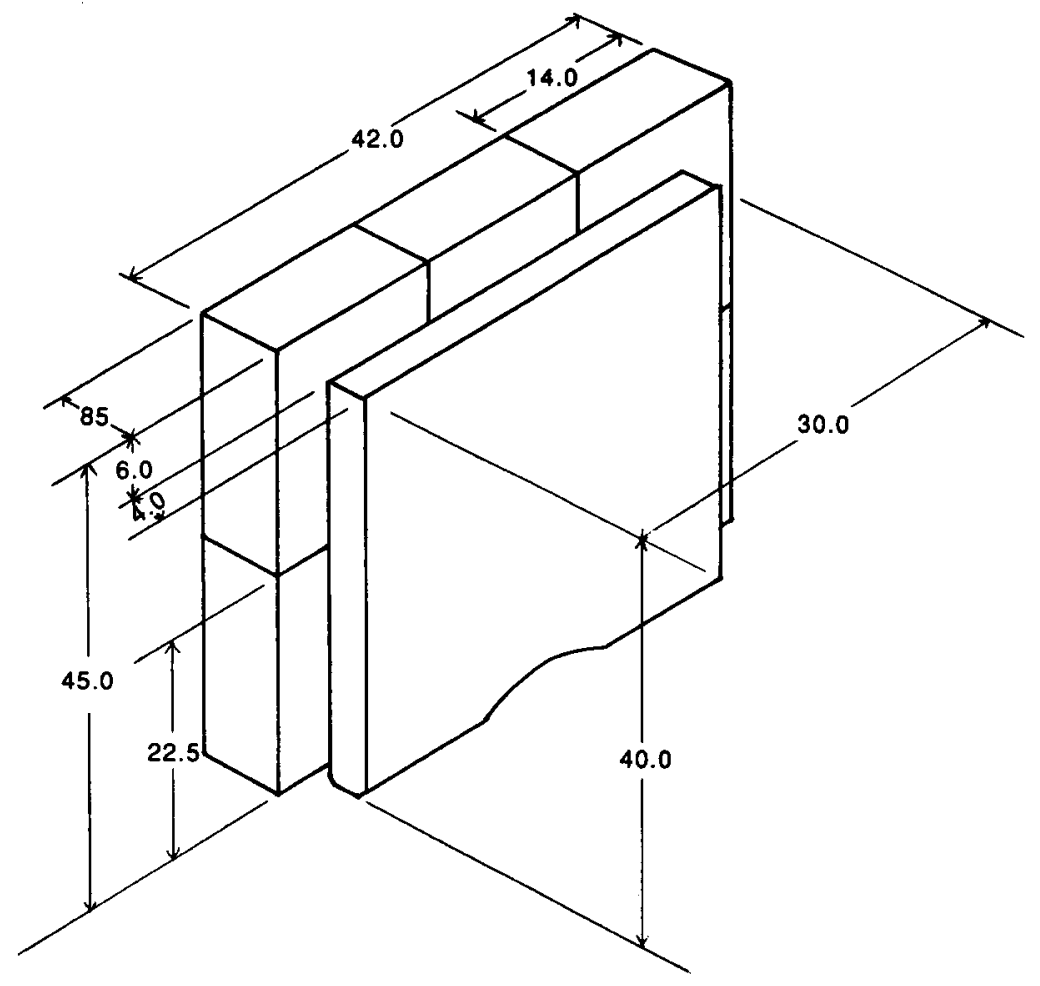

Fig. 15. A quarter period of the undulator.

is found. Small residual deviations of the field have been corrected by little shims on the poles of the undulator.

The measurements on the final undulator are shown in Fig. 16. Figures 16(a) and (b) show respectively the first and second integral of the undulator field, while Figs 16(c) and (d) show respectively the first and second integral of the field in the direction perpendicular to the undulator field. This field of course is some orders of magnitude lower.

From those measurements also the spread in the wavelength of the undulator can be found. The result is shown in Fig. 17. The average spread appeared to be $0.7 \%$. Also the fluctuation of the first integral can be determined. That result can be found in Fig. 18. The average spread appeared to be $3 \%$. The spread in the undulator field itself is much lower.

\section{$R F$ power system}

The low-level RF power signal is provided by a $40.625 \mathrm{MHz}$ crystal oscillator. In a frequency multiplier this signal is converted to $1.3 \mathrm{GHz}$. This signal is amplified by two solid state and a triode

Table 6. Design parameters TEUFEL

\begin{tabular}{lc}
\hline Design parameters & [mm] \\
\hline Undulator wavelength & 25 \\
Half gap & 4.0 \\
Overhang of magnet & 6.0 \\
Half thickness of pole & 2.00 \\
Half thickness of magnet & 4.25 \\
Height of pole & 40.0 \\
Height of magnet & 45.0 \\
Half width of pole & 15.0 \\
Half width of magnet & 21.0 \\
\hline
\end{tabular}




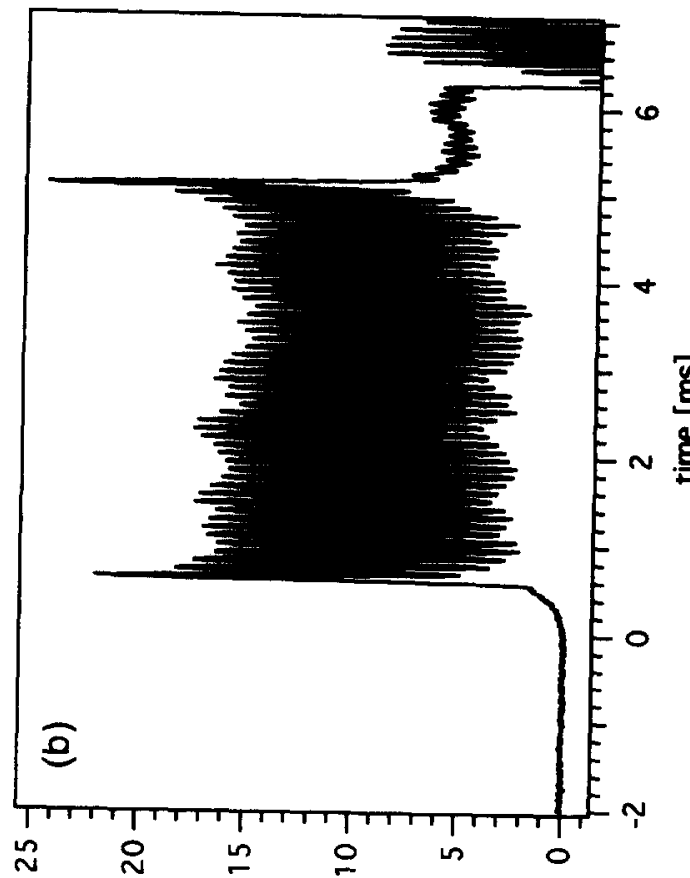

[st!un que $\left.{ }_{\varepsilon^{-}} 01 x\right] z_{2} p^{x} g \int$

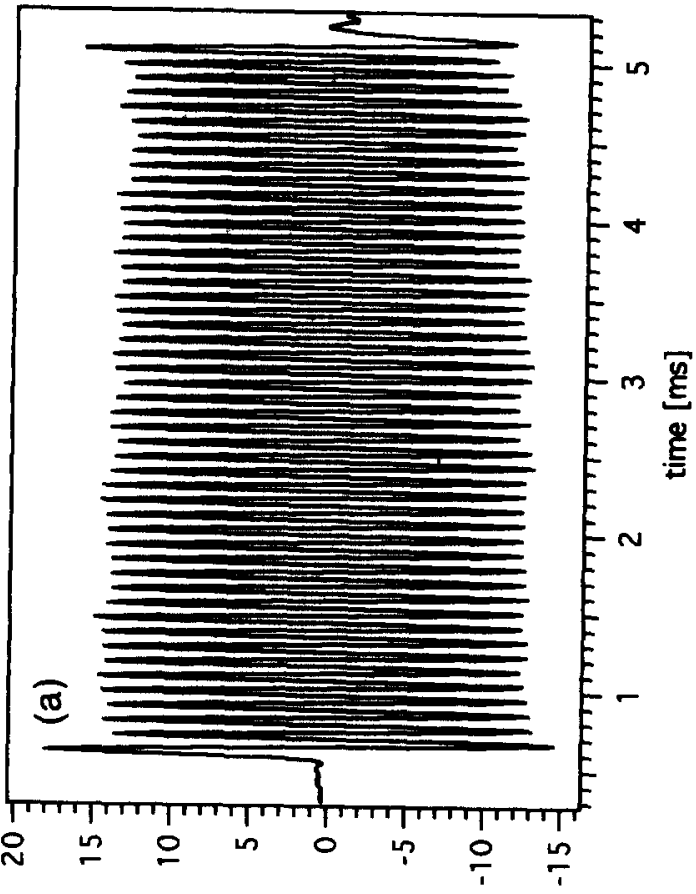

[sy!un que $\left.{ }_{\varepsilon-} O L x\right] \mathrm{zp}^{\kappa} g$ gl

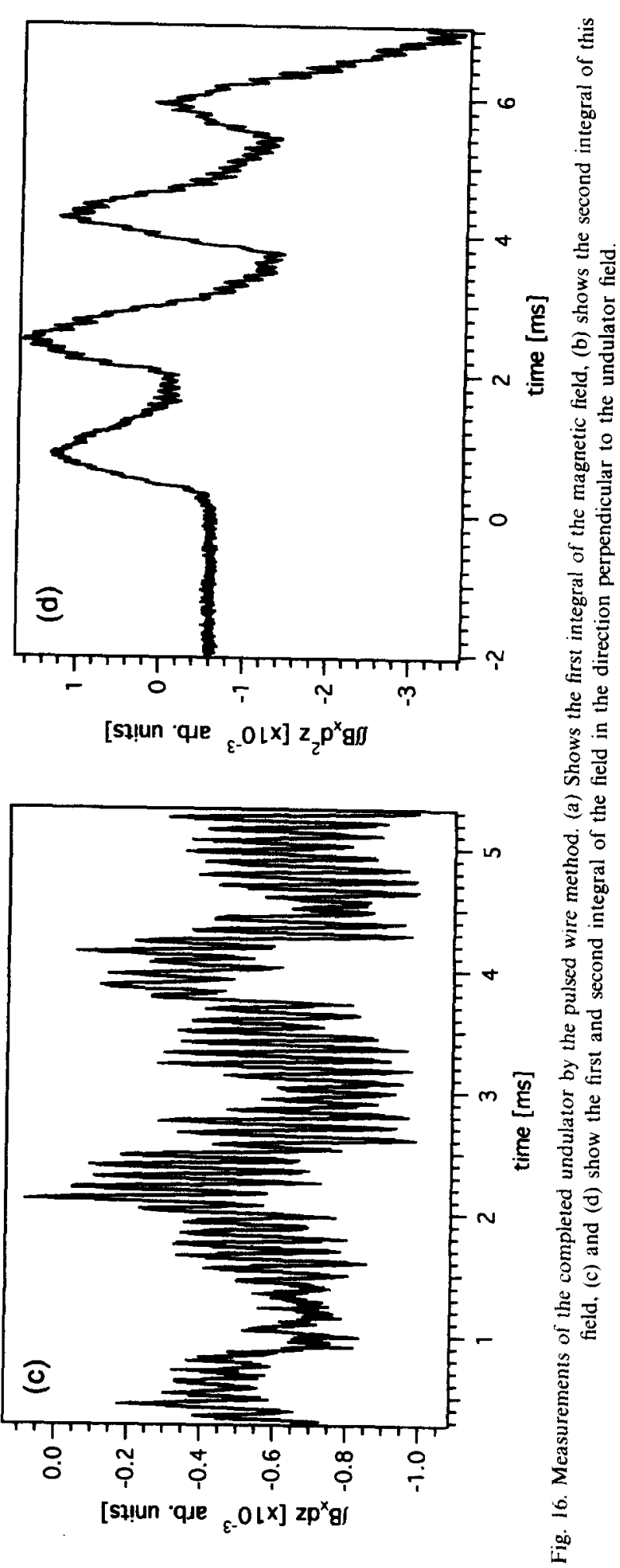




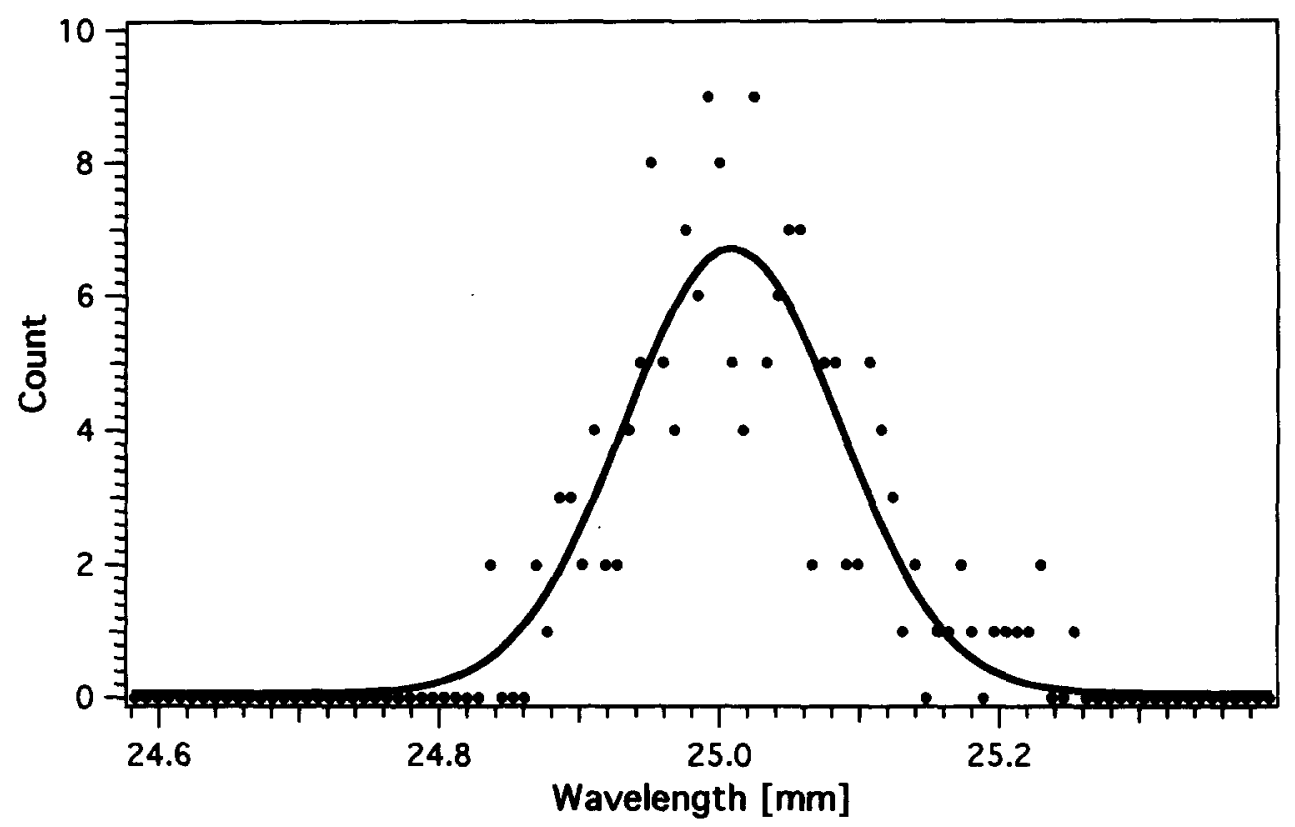

Fig. 17. A plot of the spread in length of the undulator periods.

amplifier to a level of $500 \mathrm{~W}$. The power amplifier is a Thomson TH2022C. It is a $1.3 \mathrm{GHz}$ klystron amplifier able to deliver $20 \mathrm{MW}$ of RF power in a pulse $20 \mu \mathrm{s}$ long. The klystron is powered by a line-type modulator consisting of 20 capacitors, $50 \mathrm{nF}$ each. The line will be loaded to $25 \mathrm{kV}$ and uptransformed to $250 \mathrm{kV}$. By varying the inductances between the capacitors the ripple on the flat top of the pulse can be minimized. The ripple can be made as low as a few tenth of a per cent during the last $15 \mu \mathrm{s}$ of the pulse. Such a flat modulator pulse is required in order to keep the accelerating field as constant as possible in amplitude as well as in phase. Another important source

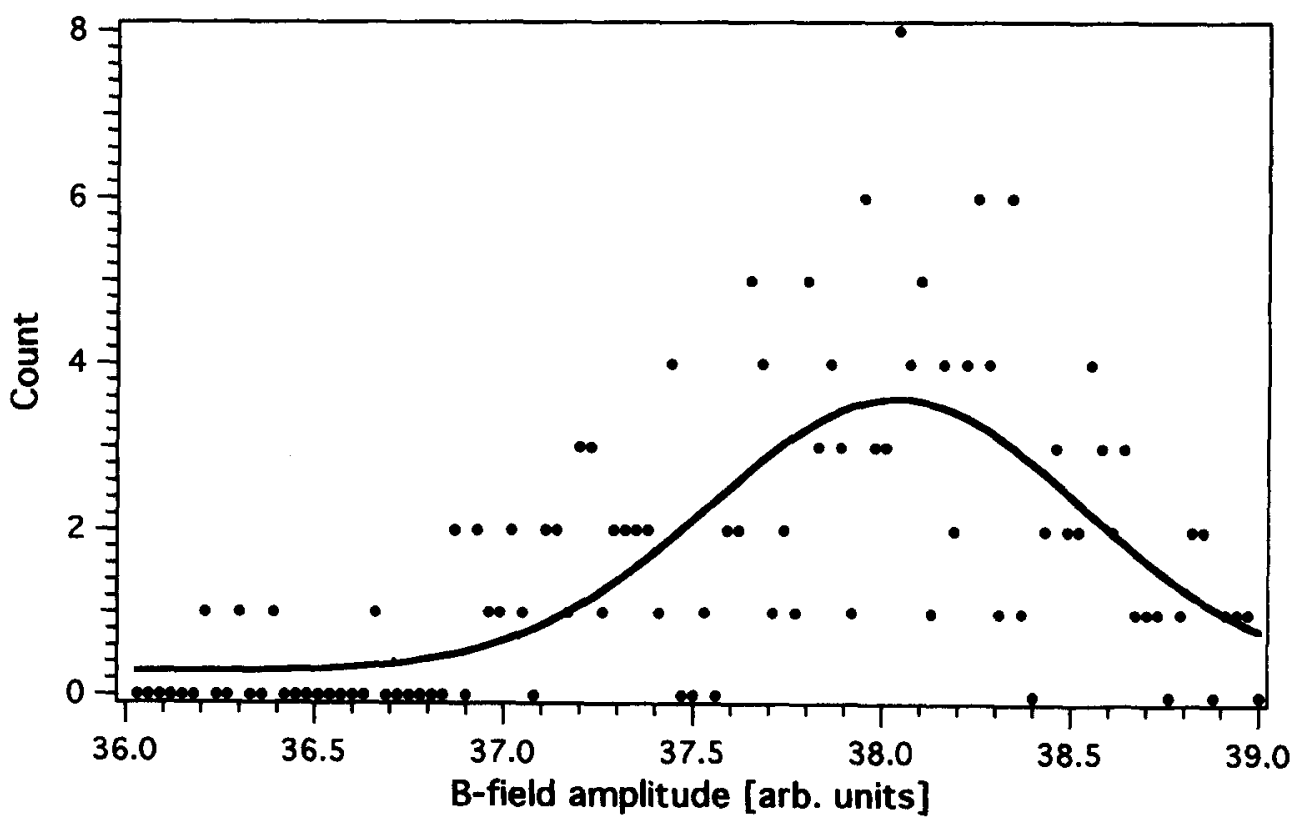

Fig. 18. A plot of the spread in amplitude of the first integral of the undulator field. 
Table 7. The TEUFEL racetrack microtron parameters

\begin{tabular}{ll}
\hline Injection energy & $6 \mathrm{MeV}$ \\
Extraction energy & $25 \mathrm{MeV}$ \\
Number of accelerations & 9 \\
Energy gain per pass & $2.11 \mathrm{MeV}$ \\
Magnetic induction & \\
$\quad$ Effective & $0.1918 \mathrm{~T}$ \\
In valley & $0.1898 \mathrm{~T}$ \\
In hill & 0.2515 \\
Drift space length & $0.9126 \mathrm{~m}$ \\
Initial harmonic number & 11 \\
Incremental harmonic number & 1 \\
Synchronous phase & $16^{\circ}$ \\
Method of beam focusing & $2-\mathrm{sector} \mathrm{AVF}$ \\
Hill-sector "amplitude" & 0.325 \\
Azimuth of valley/hill edge & $63^{\circ}$ \\
Magnet tilt angle & $6^{\circ}$ \\
Orbit separation & $7.0 \mathrm{~cm}$ \\
Angle of extraction beam & $12^{\circ}$ \\
Size of one dipole magnet & $140 \times 50 \times 35 \mathrm{~cm}^{3}$ \\
Mass of one magnet & $1700 \mathrm{~kg}$ \\
Air gap distance & \\
In valley & $50 \mathrm{~mm}$ \\
In hill & $37.74 \mathrm{~mm}$ \\
Of clamps & $55 \mathrm{~mm}$ \\
\hline
\end{tabular}

of fluctuations of the accelerating field is beam loading. The influence of beam loading is reduced by an RF feedback system as well as a feed forward system. This last system measures the effect of beam loading and creates a correction signal which will be superimposed on the original input signal and used for subsequent pulses.

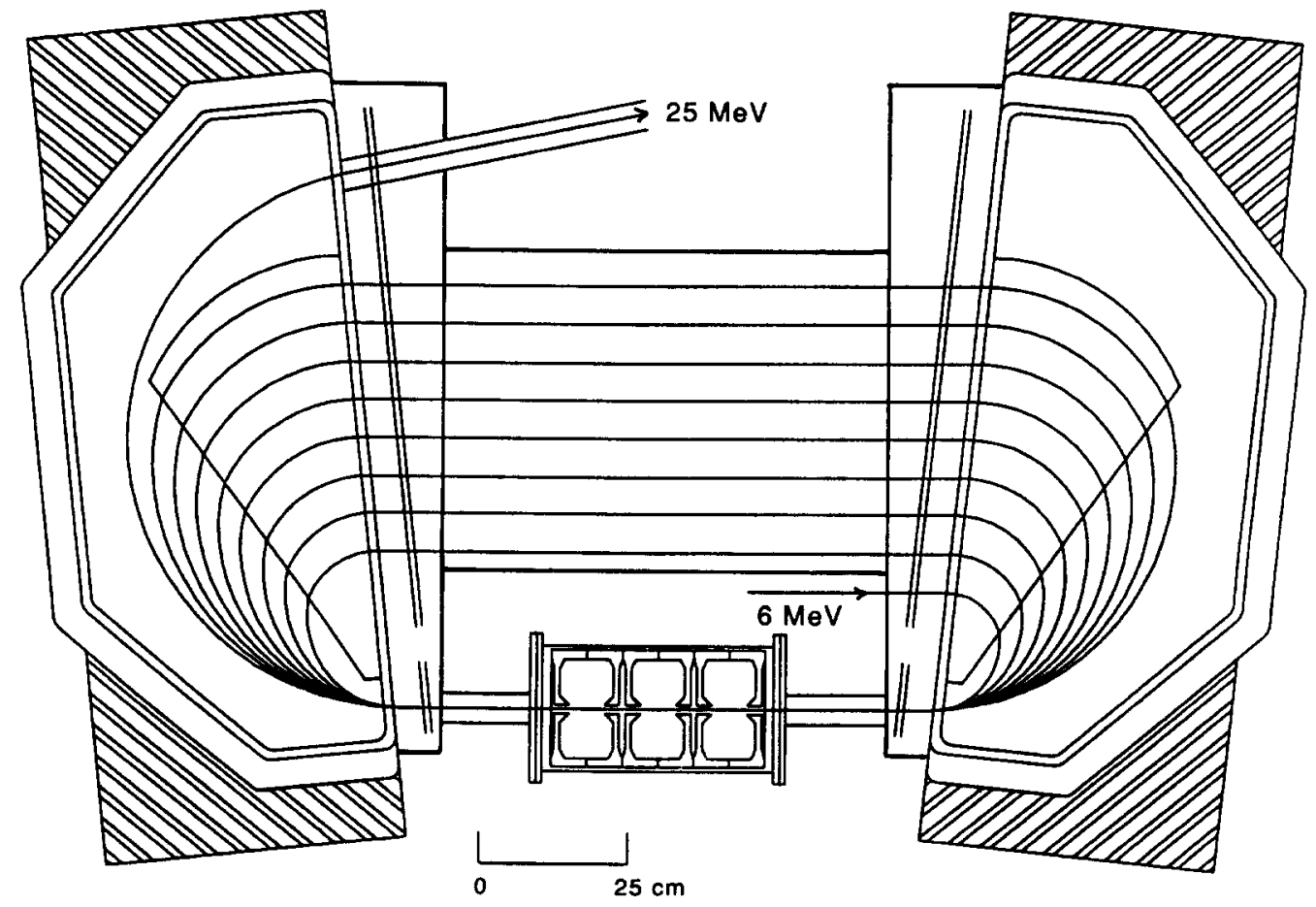

Fig. 19. On-scale median plane view of the TEU-FEL racetrack microtron. The picture shows the beam paths (including injection and extraction), the tilted two-sector bending magnets, the $\mathrm{V}$-shaped hill regions, the D-shaped coils, the active and passive clamps, the three-cell cavity, the central vacuum chamber (containing the central corrector magnet, beam position monitors, and the vacuum pump opening) and the wedgeshaped vacuum chambers. 


\section{The racetrack microtron}

Free electron lasers call for excellent properties of the driving electron beam. In this section we elaborate on racetrack microtrons (RTM) as electron sources for an FEL, in particular on the 6-25 MeV RTM of the TEU-FEL project. The choice for the RTM for this project was also made on account of the potentially high power efficiency relative to other accelerators, in particular to linacs:

-the intrinsically high beam quality of the microtron means a high gain of the FEL.

-the multiple use of the cavity structure in the RTM provides reduced structure losses of the electrical power (for the TEU-FEL project about a factor of 10 with respect to a single pass linac).

The microtron has been described in many papers and in several books [see e.g. Kapitza and Melkhin $\left.^{(3)}\right]$. Electrons are synchronously accelerated in successively larger orbits. For the RTM two
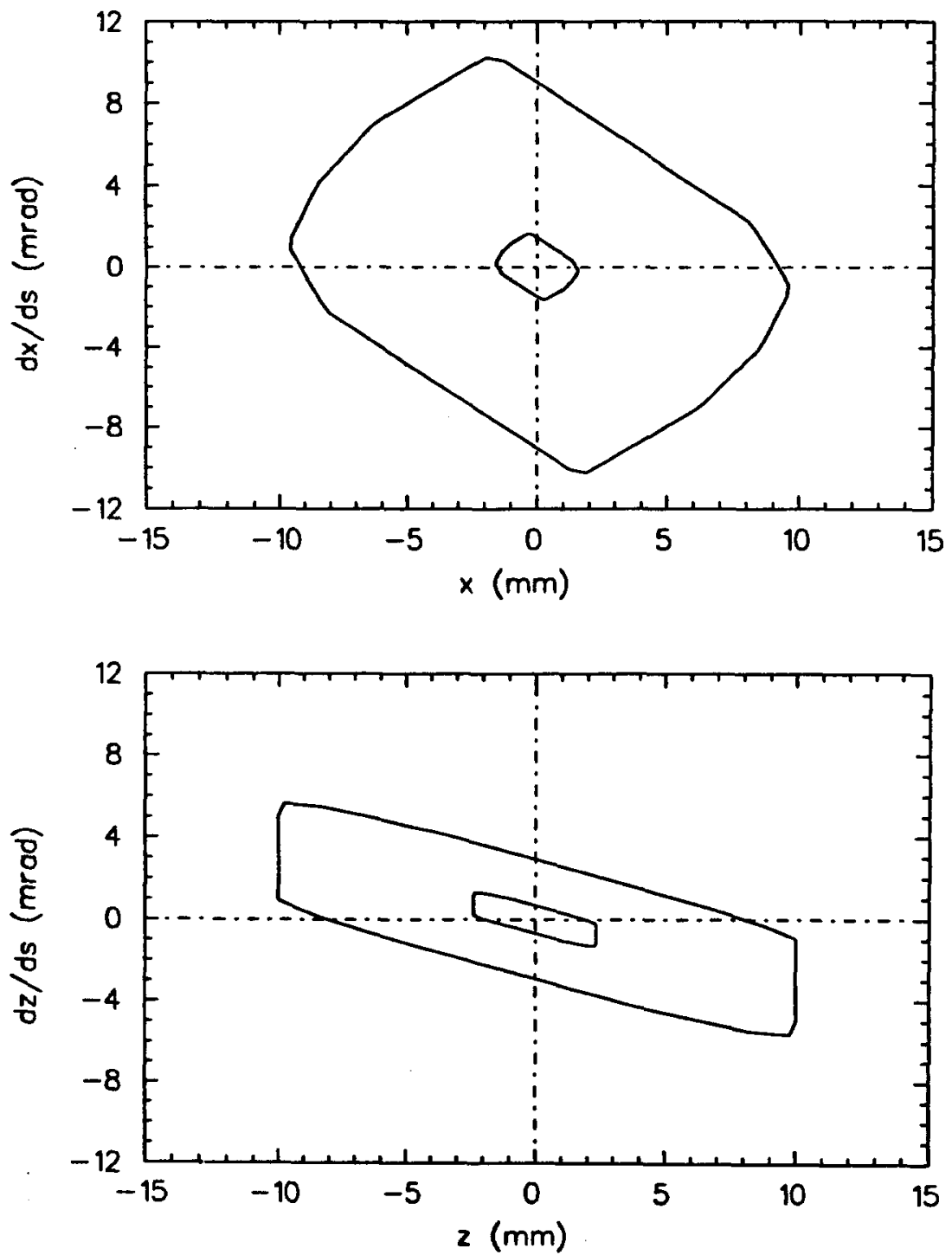

Fig. 20. Horizontal (top) and vertical (bottom) acceptance graphs of the TEU-FEL RTM. Outer curves: acceptance based on a half-aperture of $10 \mathrm{~mm}$; inner curves: emittance as originating from injection photo-cathode linac. 
conditions must be fulfilled: the length of the first complete orbit must be an integer $\mu$ (the "initial harmonic number") times the $R F$ wavelength $\left(\lambda_{R F}\right)$ and secondly the difference in path length between successive orbits must be constant, namely an integer ("incremental harmonic number" $v$ ) times $\lambda_{R F}$. For "small" microtrons usually $v$ is 1 or 2 . For ultrarelativistic particles these conditions can be translated into the following equations, relating the energy gain per turn $\Delta T$ for the synchronous particle with the average magnetic field $\mathbf{B}$, and the required injection energy $T_{0}$ with the separation distance $L$ between the two magnets constituting the RTM.

$$
v=\left(B_{c} / B\right) \Delta \gamma, \mu=\left(B_{r} / B\right) \gamma_{0}+2\left(L / \lambda_{R F}\right),
$$

where the relativistic factors $\gamma_{0}$ and $\Delta \gamma$ are given by $\gamma_{0}=1+T_{0} / E_{0}$, and $\Delta \gamma=\Delta T / E_{0}$, with $E_{0}$ the electron rest energy, and where $B_{c}$ is the "cyclotron field", $B_{\mathrm{c}}=2 \pi \mathrm{E}_{0} /\left(e c \lambda_{R F}\right)=46 \mathrm{mT}$, with $e$ the electron charge and $c$ the velocity of light. Table 7 gives the main TEU-FEL microtron parameters based on these equations, and using an $R F$ frequency of $1.3 \mathrm{GHz}$. Figure 19 gives a median plane view of the machine and of the orbit pattern.

The accelerating cavity provides a maximum accelerating gradient of $20 \mathrm{MV} / \mathrm{m}$ in a structure of $42.5 \mathrm{~cm}$ long. The macropulse beam power for a peak current of $50 \mathrm{~A}$ in the micropulses is $3 \mathrm{MW}$; the macropulse power loss in the $R F$ structure is then $0.5 \mathrm{MW}$, which means an average power dissipation of only $50 \mathrm{~W}$.

A notable feature in the TEU-FEL RTM is the fact that the left and right magnets have a small horizontal angle with respect to each other. In contrast to other RTM's, having a high degree of field uniformity $\left(1: 10^{5}\right)$ and magnets facing each other parallelly, in this RTM an azimuthally varying field profile has been incorporated, which provides extra horizontal and vertical orbit focusing, as well as isochronism. As a result the magnets must have the small tilt angle mentioned. ${ }^{(4)}$ The extra focusing is deliberately introduced to handle the large electron currents necessary to drive an FEL. The enhanced focusing implies a large horizontal and vertical acceptance area in the transverse phase planes, sufficiently to accommodate the incoming beam from the injector linac, see Fig. 20. The beam size amounts to maximum 1.5, respectively, $2 \mathrm{~mm}$ for horizontal and vertical motion. On the other hand, the inherent longitudinal focusing effect, typical for the microtron, can be observed in Fig. 21, which shows the longitudinal acceptance of the TEU-FEL RTM: the accepted energy deviation is smaller than $\pm 0.1 \mathrm{MeV}$, and the accepted phase deviation is smaller than $\pm 10^{\circ}$ ( $\pm 6 \mathrm{~mm}$ pulse length). The absolute energy and phase spread remain about constant during acceleration in the RTM.

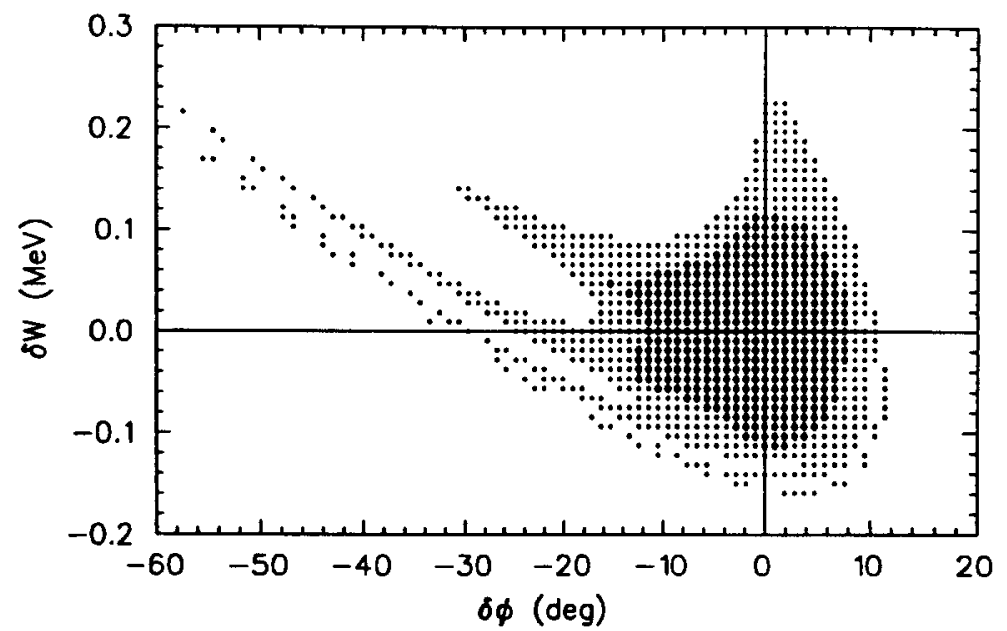

Fig. 21. Longitudinal acceptance of the TEU-FEL racetrack microtron. Inner region (large diamonds only): "asymptotical" acceptance, based on a large number of revolutions; outer and inner region together (large and small diamonds): actual acceptance of nine revolutions. 
Acknowledgements - Sponsored by Nederlands Centrum voor laser Research (NCLR), the Dutch Foundation for Technological Research (STW) and the Dutch Foundation for Research on Matter (FOM).

\section{REFERENCES}

1. D. von der Linde, Appl. Phys. B39, 201 (1986).

2. A. H. Sommer, Photo Emissive Materials. Wiley, New York (1968).

3. S. P. Kapitza and V. N. Melkhin, The Microtron. Harvard Academic, Cambridge, MA (1978).

4. J. L. Delhez and W. J. G. M. Kleeven, Part. Acc, 42, 101 (1993). 